\title{
NEURAL NETWORK BASED MIN-MAX PREDICTIVE CONTROL. APPLICATION TO A HEAT EXCHANGER
}

\author{
D.R. Ramírez M.R. Arahal E.F. Camacho
}

\author{
Departamento de Ingeniería de Sistemas y Automática, \\ Universidad de Sevilla \\ Escuela Superior de Ingenieros \\ Camino de los Descubrimientos $s / n 41092$ Sevilla \\ Phone: +34954487357 Fax: +34954487340 \\ email: $\{$ danirr, arahal,eduardo\}@cartuja.us.es
}

\begin{abstract}
Min-max model predictive controllers (MMMPC) have been proposed for the control of linear plants subject to bounded uncertainties. The implementation of MMMPC suffers a large computational burden due to the numerical optimization problem that has to be solved at every sampling time. This fact severely limits the class of processes in which this control is suitable. In this paper the use of a Neural Network (NN) to approximate the solution of the min-max problem is proposed. The number of inputs of the NN is determined by the order and time delay of the model together with the control horizon. For large time delays the number of inputs can be prohibitive. A modification to the basic formulation is proposed in order to avoid this later problem. Simulation and experimental results are given using a heat exchanger. Copyright 2001 IFAC
\end{abstract}

Keywords: Predictive control, Minimax techniques, Neural networks, Uncertain linear systems, Process control.

\section{INTRODUCTION}

Mathematical models and specially control models, which have to be kept simple, can only describe the dynamics of the process in an approximately way. Most of the approaches used for modeling uncertainties assume that there is a family of models and that the plant can be described exactly by one of the models belonging to the family. The approach considered here is global uncertainties. In this way, uncertainties will be considered to affect the 1-step ahead prediction equation, i.e. the uncertainties will affect the prediction capability of the model. The global uncertainties approach can be found in (Camacho and Bordons, 1999). A robust adaptive Min-Max MPC controller has been applied to a solar power plant in (Camacho and Berenguel, 1997).
Min-Max control techniques have in common a great computational burden which limits the range of process to which can be applied to those with slow dynamics. Neural Networks have been used with success in Model Predictive Control (Arahal et al., 1998). In this paper we present a way to implement Min-Max Predictive Controllers for linear plants using a Neural min-max solver.

Another difficult arises when the plant has a large dead time. In this case we have to take into account the effect of past values of the control signal in the min-max optimization. We show here how to overcome this problem without loosing the robustness properties of Min-Max MPC and with only a gain of conservativeness in the control law.

The paper is organized as follows: section 2 presents the basic Min-Max MPC with bounded 
global uncertainties algorithm. Next the Neural min-max solver for plants without large delays is introduced. Section 3 is devoted to the MinMax MPC with bounded global uncertainties algorithm for plants with large delays. Section 5 shows the application of the NN based MMMPC to a heat exchanger and section 6 presents the conclusions.

\section{MIN-MAX MPC WITH GLOBAL LNCERTAINTIES}

The objective of MPC control is to compute the future control sequence $u(t), u(t+1), \ldots, u(t+N-$ 1) in such a way that the optimal j-step ahead predictions $y(t+j \mid t)$ are driven close to the set point sequence $w(t), w(t+1), \ldots, w(t+N-1)$ for the prediction horizon. The way in which the system will approach the desired trajectories will be indicated by a cost function $J$ which depends on the presents and future control signals and uncertainties.

When bounded uncertainties are considered explicitly, it would seem that a more robust control would be obtained if the controller tried to minimize the objective function $J$ for the worst situation. That is, by solving the following minmax problem:

$$
\min _{u \in U} \max _{\theta \in \Theta} J(\theta, u)=\min _{u \in U} J^{*}(u)
$$

where $\theta$ represents the sequence of future uncertainties and:

$$
J^{*}(u)=\max _{\theta \in \Theta} J(\theta, u)
$$

being $\Theta=\{\theta / \underline{\theta} \leq \theta \leq \bar{\theta}\}$. The function to be minimized $J^{*}(u)$ is the maximum of the objective function $J(\theta, u)$ which measures how well the process output follows the reference trajectories.

Min-Max MPC can be formulated either in state space or using input-output description. The latter is used here since process with large dead time are easy to describe using transfer function representations.

The most usual form of $J(\theta, u)$ is a quadratic criterion:

$$
\begin{aligned}
J(\theta, u) & =\sum_{j=N_{\mathrm{L}}}^{N_{2}}\left(y_{k+j \mid k}-w_{k+j}\right)^{2} \\
& +\lambda \sum_{j=1}^{N_{\mathrm{u}}}\left(\Delta u_{k+j-1}\right)^{2}
\end{aligned}
$$

where: $\Delta=1-z^{-1}, N_{1}$ and $N_{2}$ define the beginning and end of the cost horizon, $N_{u}$ is the control horizon and $y_{k+j \mid k}$ is the prediction output. Other types of objective functions have been used in literature. In (Campo and Morari, 1987) a $\infty-\infty$ norm is used while in (Allwright, 1994) a $1-\infty$ norm is proposed.

When a global uncertainties approach is used the way of modeling the uncertainties is to assume that all modeling errors are globalized in a vector of parameters. such that the plant can be described by the following family of models:

$$
\dot{y}(t+1)=\dot{f}(y(t), u(t))+\theta(t)
$$

In (Camacho and Bordons, 1999) is shown that global uncertainties can be related to other types of uncertainties. When the model considered in (4) is linear it is common to choose a CARIMA model as prediction model (Camacho and Bordons, 1999).

The error concept present in a CARIMA model can be extended to consider explicitly the disturbances in the prediction model yielding:

$$
\tilde{A}\left(z^{-1}\right) y(t)=z^{-d} B\left(z^{-1}\right) \Delta u(t-1)+\theta(t)
$$

where $\dot{A}\left(z^{-1}\right)=\Delta A\left(z^{-1}\right)$ and $\theta(t) \in \Theta$. This prediction model will be referred as an integrated uncertainties prediction model ((Camacho and Bordons, 1999)).

It is easy to see that the prediction model is an affine function of $\theta(t)$, which in turn leads to a function $J(\theta, u)$ that is convex in the hypercube of $\theta(t)$. That implies that the maximum of $J(\theta, u)$ is reached on one of the vertices of $\Theta$. Also the maximum is unique and it can be easily seen that function $J^{*}(u)$ is a piecewise quadratic function of $u$ (Camacho and Bordons, 1999).

Using an integrated uncertainties prediction model allows to reject step disturbances but produces a continuos grow of the uncertainty band (Camacho and Bordons, 1999) and (Ramírez and Camacho, 2000). To have stable uncertainty bands while keeping the step response rejection the following constraints to optimization have to be added (Ramírez and Camacho, 2000):

$$
[\underline{\Theta} \ldots \underline{\Theta}]^{T} \leq T\left[\theta(t+1) \ldots \theta\left(t+N_{2}\right)\right]^{T} \leq[\bar{\Theta} \ldots \bar{\Theta}]^{T}
$$

where $T$ is a lower triangular matrix of appropriate dimension.

\section{WORST CASE PREDICTION OF DELAYED OUTPUTS MIN-MAX MPC}

The strategy used here to apply the Min-Max MPC to process with large dead time splits the problem in two stages: 
- Estimation of process output after the dead time. This is a prediction problem.

- Min-Max MPC control law calculation for the process after the dead time, taking into account the uncertainty in the estimation of the process output up to the dead time. This is a control design problem.

This strategy applied for a first order CARIMA prediction model can be formulated as the following steps:

- First, compute the bounds for $y(k+d)$ and $y(k+d-1)$

$$
\begin{aligned}
\bar{\theta}_{k+d-1}^{\prime} & =\max _{\theta}\left(y_{k+d-1}-\hat{y}_{k+d-1}\right) \\
\underline{\theta}_{k+d-1}^{\prime} & =\min _{\theta}\left(y_{k+d-1}-\hat{y}_{k+d-1}\right) \\
\bar{\theta}_{k+d}^{\prime} & =\max _{\theta}\left(y_{k+d}-\dot{y}_{k+d}\right) \\
\underline{\theta}_{k+d}^{\prime} & =\min _{\theta}\left(y_{k+d}-\tilde{y}_{k+d}\right)
\end{aligned}
$$

where $\hat{y}_{k+d-1}$ y $\hat{y}_{k+d}$ are nominal values for $y_{k+d-1}$ and $y_{k+d}$.

- Second, obtain the control law solving the min-max problem for the nominal plant without dead time using the following functional:

$$
f\left(y_{k+d}, y_{k+d-1}\right)=\min _{\Delta u} \max _{\theta_{k+d}^{\prime}, \theta} J\left(\Delta u, \theta_{k+d}^{\prime}, \theta\right)
$$

taking into account the uncertainties in the plant output for $y(k+d)$ and $y(k+d-$ $1)$, which have to be reflected in the future output predictions. Thus, the future output predicted will be:

$$
\begin{aligned}
y_{k+d+1}= & (1+a)\left(y_{k+d}+\theta_{k+d}^{\prime}\right)-a\left(y_{k+d-1}\right. \\
& \left.+\theta_{k+d-1}^{\prime}\right)+\theta_{k+d+1} \\
y_{k+d+2}= & (1+a) y_{k+d+1}-a\left(y_{k+d}+\theta_{k+d}^{\prime}\right) \\
& +\theta_{k+d+2}
\end{aligned}
$$

It can be seen that the prediction model is an affine function of $\theta_{k+d-1}^{\prime}, \theta_{k+d}^{\prime}$ as well of $\theta$. This implies that the maximum of $J$ for $\theta_{k+d-1}^{\prime}, \theta_{k+d}^{\prime}$ will be reach in one of the vertexes of the hypercube $\left\{\theta_{k+d-1}^{\prime}, \theta_{k+d}^{\prime}\right\}$. The computational burden increment is the the same to an increment of two in the prediction horizon.

This strategy is more conservative than a traditional Min-Max MPC in the sense that only the maximum and minimum values for $y(k+d)$ and $y(k+d-1)$ are considered as starting points to the min-max optimization. To illustrate this point consider a process with a dead time of $d=6$. In a traditional Min-Max MPC there is 32 different possible values for $y_{k+d-1}$ and 64 for $y_{k+d}$, which will be used as starting point for the rest of the optimization. With the strategy presented here only two possible values for each of them are considered, which yields 4 combinations of the most extreme values of $y_{k+d-1}$ and $y_{k+d}$. In addition two of the combinations are unrealistic, because there is no way to get the plant outputs equal to the nominal values of $y_{k+d-1}$ and $y_{k+d}$ plus $\left\{\underline{\theta}_{k+d-1}^{\prime}, \bar{\theta}_{k+d}^{\prime}\right\}$ or $\left\{\vec{\theta}_{k+d-1}^{\prime}, \underline{\theta}_{k+d}^{\prime}\right\}$. Finally, two sources of uncertainty are considered in the prediction of the $y_{k+d+1}$ and $y_{k+d+2}$, i.e. the global uncertainty $\theta(t)$ and the bounds for $y_{k+d-1}$ and $y_{k+d}$.

To avoid this excess of conservatism only the combinations $\left\{\underline{\theta}_{k+d-1}^{\prime}, \underline{\theta}_{k+d}^{\prime}\right\}$ and $\left\{\bar{\theta}_{k+d-1}^{\prime}, \bar{\theta}_{k+d}^{\prime}\right\}$ are considered in the optimization which has the added benefit of halving the amount of vertexes to be considered to compute the max part of the min-max problem.

The amount of time required to compute the control sequence can be further reduced by considering that for linear prediction models the superposition principle holds, hence the part of the output prediction due to the uncertainty $\theta(t)$ can be separated from the part due to control actions. It can be easily seen that the bounds values $\left\{\underline{\theta}_{k+d-1}^{\prime}, \underline{\theta}_{k+d}^{\prime}\right\}$ and $\left\{\bar{\theta}_{k+d-1}^{\prime}, \bar{\theta}_{k+d}^{\prime}\right\}$ are independent of the values of $\left\{y_{k-1}, y_{k}\right\}$ and are also independent of the past and future control actions. This considerations imply that the bounds for $y(k+d)$ and $y(k+d-1)$ have only to be computed once.

Figure 1 illustrate the behaviour of a Min-Max MPC controller using this strategy compared with a traditional Min-Max MPC controller and a conventional MPC controller, using the same values for control and prediction horizons and control effort weighting. For the Min-Max MPC controllers the bounds for the uncertainty were $\underline{\theta}=\underline{\theta}=-0.5$ and $\bar{\theta}=\bar{\Theta}=0.5$. The faster response over the traditional Min-Max MPC is due to the greater conservativeness of considering only the maximum and minimum values of $y_{k+d-1}$ and $y_{k+d}$.

Finally, it is noteworthy that this scheme is suitable for other implementations of Min-Max MPC even for the numerical one, with the benefit a reduced time of computation of the min-max solution.

\section{NEURAL MIN-MAX SOLVER}

Artificial Neural Networks have been used with success in predictive control and identification (Arahal et al., 1998), especially when non linear systems are considered. When linear systems without taking into account uncertainties are considered there is no need to use a non linear estimation method like Neural Networks because 


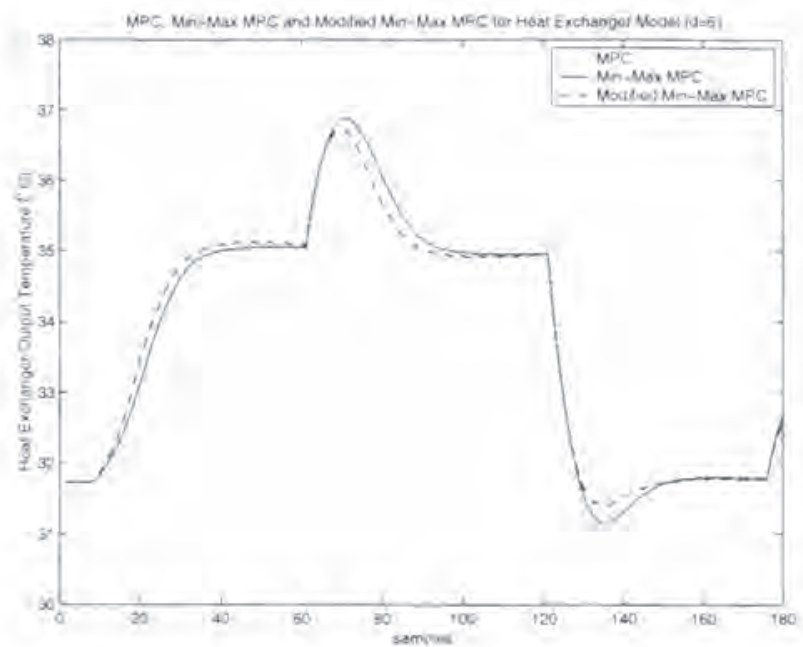

Fig. 1. Output of a MPC, Min-Max MPC and Min-Max MPC modified controllers applied to the plant $y_{k+1}=0.9048 y_{k}+0.0128 u_{k-6}$.

efficient solutions exist in the form of closed formulae (for the unconstrained case) or mathematical programming (constrained case, see (Camacho and Bordons, 1999)). However if uncertainties are taking into account there is no efficient numerical methods to handle problems like (1). In this case Neural Networks can be a good choice to apply this strategy to process with reasonably fast $d y$ namics.

The solution of the min-max problem can be precomputed for a number useful situations. A NN can be used to approximate function $J^{*}$ in (2) with mild assumptions about the smoothness of the approximated mapping. If this is done properly, then solving the min-max problem would only amount to just query the NN for an output $u=N N(\mathbf{x})$ to current input $\mathbf{x}$ where $\mathbf{x}$ is the process state and set point. This allows the MinMax MPC use in real time in a larger class of process.

Here we concentrate in a neural min-max solver for a first order plant using an integrated uncertainties prediction model:

$$
y_{k+1}=(1+a) y_{k}-a y_{k-1}+b \Delta u_{k}+\theta
$$

The function min-max to be approximated. which will take the set point and the actual and past yalues of process output as arguments, depends on the parameters of the plant as well of the parameters of the controller.

Figure 2 shows the approximation error using a Multi Layer Perceptron (MLP) with 10 neurons in the hidden layer when plant model parameters are $a=b=0.25$ and controller parameters are $N_{u}=4 . N_{2}=8 \lambda=0 . \bar{y}, \bar{\theta}=0.1, \underline{\theta}=-0.1$ and the set point is 0.25 . A set of patterns distributed on an uniform grid with a spacing of 0.05 , making a total of 1681 patterns, was built. The MLP was

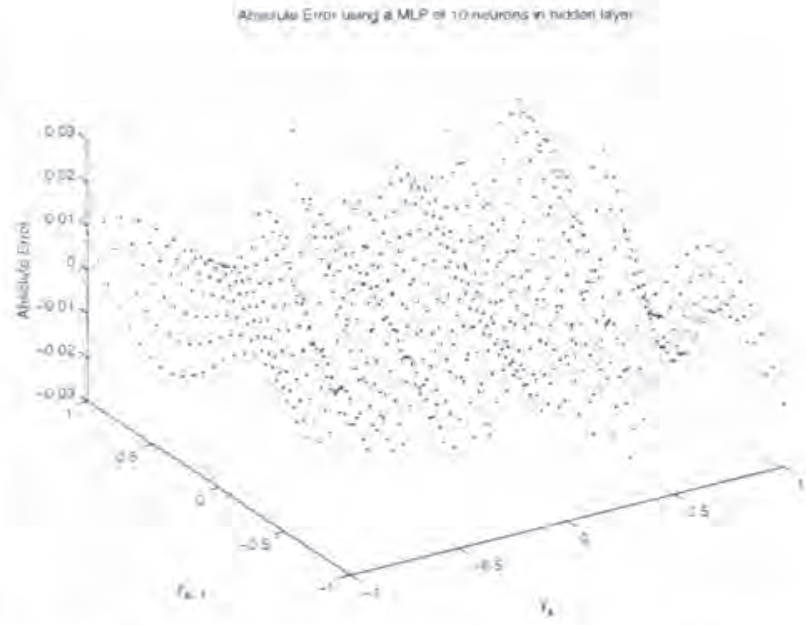

Fig. 2. Approximation error using a MLP with 10 neurons in hidden layer for a controller with $N_{u}=4 . N_{2}=8 \lambda=0.5, \bar{\theta}=0.1, \underline{\theta}=-0.1$

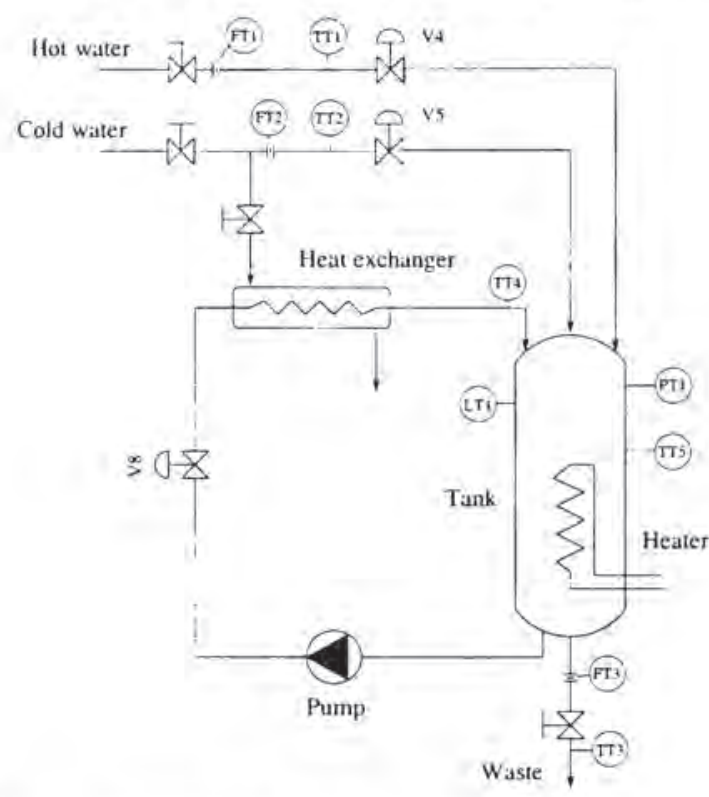

Fig. 3. Diagram of the Pilot Plant

trained using the Levenberg-Marquardt method and a final sum of squared errors of 0.105 was obtained. The trained MLP was used with a different set of patterns built with a spacing of 0.08 . and the errors obtained are shown in figure 2 . The magnitude of errors is small enough to allow its real time use as shown for the heat exchanger in section 5 .

\section{APPLICATION TO A HEAT EXCHANGER}

The controller presented in sections 4 and 3 has been applied to a heat exchanger which is part of a pilot plant. A diagram of the pilot plant which shows its main elements as well as the localization of the various instruments is given in Figure 3.

The main elements are: 
- Feed circuit. The plant has two input pipes, a cold water one (which temperature is regulated by a cooling plant) and a hot water one (at about $70^{\circ} \mathrm{C}$ ). The temperatures and the flows of the inputs are measured with thermocouples and orifice plates respectively. with controlled pneumatic valves for regulating the input flows.

- Tank. It has a height of $1 \mathrm{~m}$ and an interior diameter of $20 \mathrm{~cm}$, it is thermically insulated, and with an approximate volume of $31 \mathrm{l}$. In its interior there is a $15 \mathrm{~kW}$ electric resistor for heating, also an overflow pipe, an output pipe and another one for recirculating the water through the exchanger.

- Recirculation circuit. The hot water in the tank can be cooled by entering cold water through the cooling circuit. This circuit is composed of a centrifugal pump that circulates the hot water from the bottom of the tank through a tube bundle heat exchanger returning at a lower temperature at its top.

The plant control elements are connected to a PMC10 unit operated under the ORSI Automazione CUBE control software. The PMC10 architecture allows to implement control algorithms programmed in a $\mathrm{PC}$ using CUBE's ITER II language. However the execution time is restricted to 100 milliseconds and taking into account that PMC10 CPU is an old Intel 8086 it is clear that the min-max problem cannot be solved numerically in the PMC10. So it is a suitable scenario to the neural network implementation described in this paper.

Several predictive control strategies has been applied to the control loop V8-TT4. In (Camacho and Bordons, 1999) a precomputed linear GPC is applied. On the other hand nonlinear predictive control has been applied to this control loop in (Ramírez et al., 1999) (in this work control actions were calculated in a PC, rather than the PMC10 unit).

A first order lineal model for the transfer function from $V 8$ to $T T 4$ has been obtained by step response. The initial conditions were $V 8=$ $50 \%, T T_{4}=31.73^{\circ} \mathrm{C}, T T 5$ controlled with a PID around $50^{\circ} \mathrm{C}$, constant tank level of $76.8 \%$ and the set point for the cooling plant to get $T T_{2}$ around $23.3^{\circ} \mathrm{C}$. A step in the aperture of valve $V 8$ from $50 \%$ to $70 \%$ yields the following model:

$$
G(s)=\frac{0.135}{6 s+1} e^{-6 s}
$$

A CARIMA model is obtained sampling model 6 with a sampling time of 1 second (giving a delay $d$ of 6 ) and the controllers parameters have been chosen to be $N_{u}=3, N_{1}=7, N_{2}=N_{\theta}=12, \lambda=$ $3.0, \bar{\theta}=0.2, \underline{\theta}=-0.2$. To train the $\mathrm{NN}$ a set of patterns were generated for set point values of $32^{\circ} \mathrm{C}, 35^{\circ} \mathrm{C}$ and $37^{\circ} \mathrm{C}$. A total of 36963 patterns were generated for the training set and 6627 for the test set. A MLP with 10 neurons in the hidden layer was trained using the Levenberg-Marquardt rule until the average error in both sets were under $1.5 \%$.

The resulting controller was applied to the heat exchanger and some of the experimental results are depicted in figures 4 to $\bar{\jmath}$.

Figure 4 shows a set point tracking experiment. It is noteworthy that the set point is higher than the operating point considered for modeling the heat exchanger dynamics. The noisy output is due to the variations in the tank temperature (TT5) which is regulated by a local PI. Also it is noteworthy that the V8 aperture is not affected by fast TT5 changes, on the other hand V8 aperture follows TT2 changes and TT5 slow changes.

Disturbance rejection is illustrated in figure 5 . In this case the manual valve of cold water was closed for 15 and 20 seconds, causing the temperature to greatly deviate from the set point. The controller reacts by closing the recirculation valve $\mathrm{V} 8$ to low the temperature by having less hot water to be cooled. Opening again the valve is another disturbance and the controller has to open again the V8 valve to bring the temperature TT 4 to the desired value.

Finally a third experiment depicting a slow variation of plant dynamics is shown in figure 5 . In this case, the tank temperature (TT5) was raised from the nominal point $\left(50^{\circ} \mathrm{C}\right)$ to a point around $70^{\circ} \mathrm{C}$. It is clear that the controller is able to keep the output close to the set point as soon as the tank temperature reaches the new operating point. Meanwhile a small offset is observed as expected, because the uncertainty grows as a ramp and the integrated uncertainties MMMPC is formulated to reject step disturbances.

\section{CONCLUSIONS}

A Neural Min-Max MPC controller has been proposed and applied to a pilot plant. Problems related to process delay have been tackled and discussed taking into account real time implementation requirements. The suitability of a NN approach has been discussed and brought to practice. Further investigations will be needed to include constraints and adaptive skills in the control strategy.

\section{REFERENCES}

Allwright, J.C. (1994). Advances in ModelBased Predictive Control. Chap. On min-max 


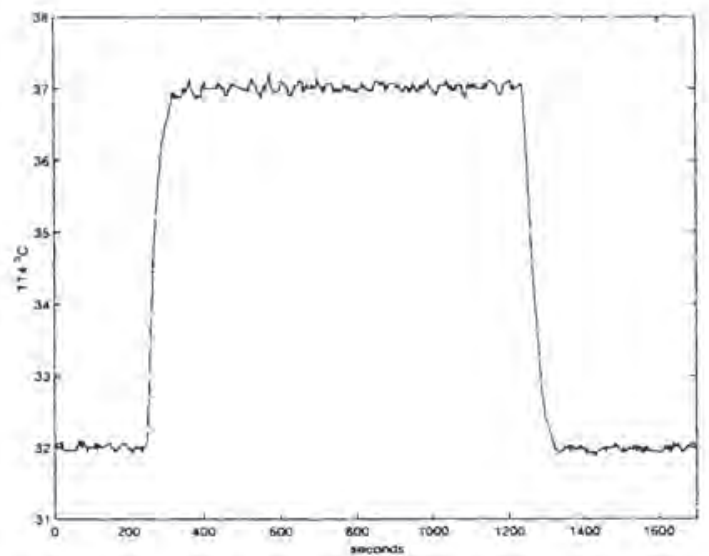

(a)
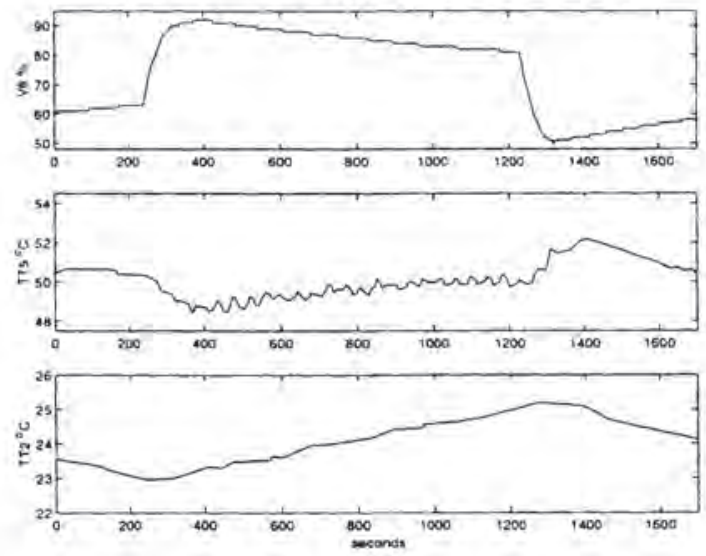

(b)

Fig. 4. Set point tracking experiment; a) set point and heat exchanger output temperature b) Valve V8 aperture, tank temperature TT5 and cold water temperature TT2

Model-Based Predictive Control. Oxford University Press.

Arahal, M.R., M. Berenguel and E.F. Camacho (1998). Neural identification applied to predictive control of a solar plant. Control Engineering Practice 6, 333-344.

Camacho, E. F. and Manuel Berenguel (1997). Robust adaptive model predictive control of a solar plant with bounded uncertainties. International Journal of Adaptive and Signal Processing 11, 311-325.

Camacho, E.F. and C. Bordons (1999). Model Predictive Control. Springer-Verlag.

Campo, P.J. and M. Morari (1987). Robust model predictive control. In: Proc. American Control Conference. pp. 1021-1026.

Ramírez, D.R. and E.F. Camacho (2000). Properties of min-max mpc with global uncertainties. Internal Report, Dept. Ingeniería de Sistemas y Automática, Universidad de Sevilla.

Ramírez, D.R., D. Limón-Marruedo, J. GómezOrtega and E.F. Camacho (1999). Model based predictive control using genetic algorithms. Application to a pilot plant. In: Proc. European Control Conference, ECC'g9.

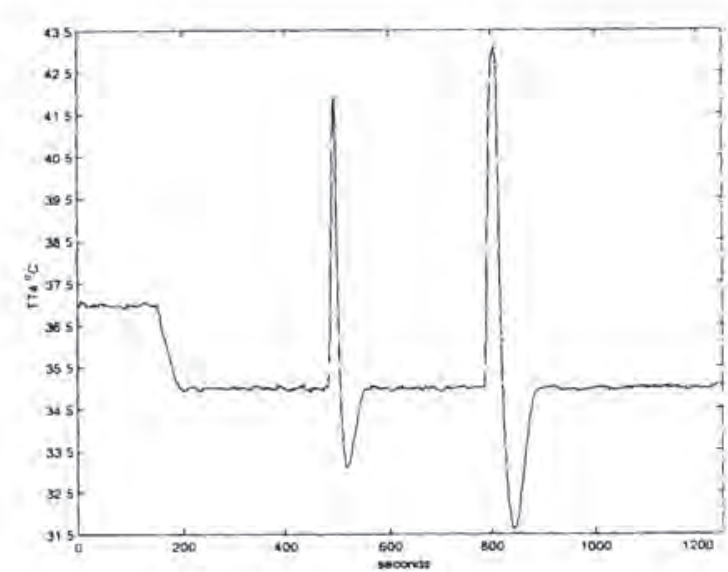

(a)
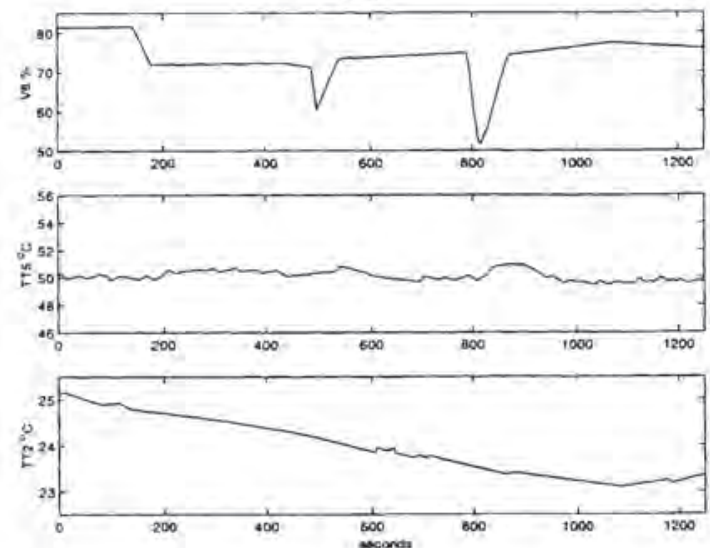

(b)

Fig. 5. Disturbance rejection experiment: a) set point and heat exchanger output temperature b) Valve V8 aperture, tank temperature TT5 and cold water temperature TT2

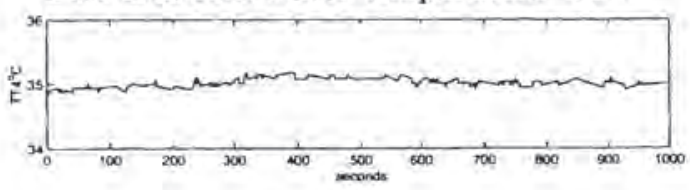

(a)
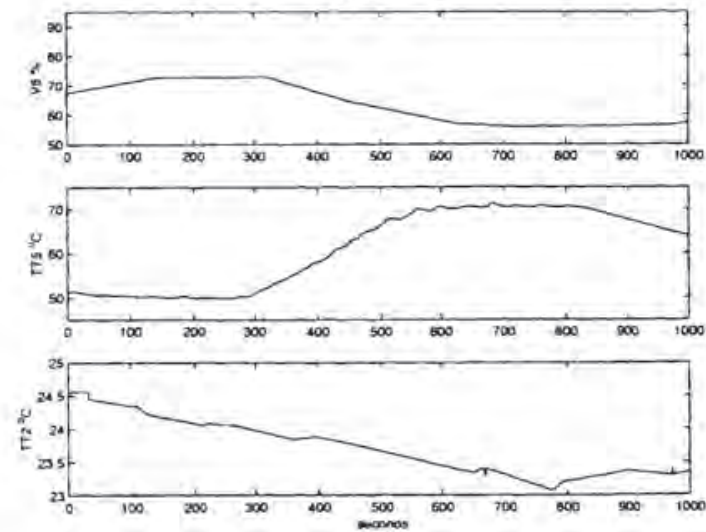

(b)

Fig. 6. Slow variation of plant dynamics experiment: a) set point and heat exchanger output temperature b) Valve V8 aperture, tank temperature TT5 and cold water temperature TT2 